\title{
Emergent Horizons in the Laboratory
}

\author{
Ralf Schützhold \\ Institut für Theoretische Physik, Technische Universität Dresden, 01062 Dresden, Germany
}

\begin{abstract}
The concept of a horizon known from general relativity describes the loss of causal connection and can be applied to non-gravitational scenarios such as out-of-equilibrium condensed-matter systems in the laboratory. This analogy facilitates the identification and theoretical study (e.g., regarding the trans-Planckian problem) and possibly the experimental verification of "exotic" effects known from gravity and cosmology, such as Hawking radiation. Furthermore, it yields a unified description and better understanding of non-equilibrium phenomena in condensed matter systems and their universal features. By means of several examples including general fluid flows, expanding BoseEinstein condensates, and dynamical quantum phase transitions, the concepts of event, particle, and apparent horizons will be discussed together with the resulting quantum effects.
\end{abstract}

\section{MOTIVATION}

Typically, the investigation of a given condensedmatter system starts with understanding its equilibrium configuration, e.g., the ground state $\left|\psi_{0}\right\rangle$ at zero temperature. However, strictly speaking, this equilibrium state provides a valid description for static situations only. A realistic laboratory system, on the other hand, is never completely static - but subject to some time-dependent external conditions such as a varying pressure or magnetic field. As long as these external variations are sufficiently slow (in comparison with the internal response time), one would argue that the equilibrium state should provide a good approximation to the real state of the system. One way of making this argument more precise is given by the adiabatic theorem: For a time-dependent quantum system at zero temperature (initially) with a discrete spectrum $H(t)\left|\psi_{n}(t)\right\rangle=E_{n}(t)\left|\psi_{n}(t)\right\rangle$, the actual quantum state $|\psi(t)\rangle$ as a solution of the Schrödinger equation $i \partial_{t}|\psi(t)\rangle=H(t)|\psi(t)\rangle$ stays close to the ground state $\left|\psi_{0}\right\rangle(\hbar=1)$

$$
|\psi(t)\rangle \approx\left|\psi_{0}(t)\right\rangle+\sum_{n>0} \frac{\left\langle\psi_{n}|\dot{H}| \psi_{0}\right\rangle}{\left(E_{n}-E_{0}\right)^{2}} e^{i \varphi_{n}}\left|\psi_{n}(t)\right\rangle
$$

provided that the external time-dependence of the Hamiltonian $\dot{H}$ is slow enough compared to the internal response times of order $\mathcal{O}\left(1 /\left[E_{n}-E_{0}\right]\right)$, i.e., $\left\langle\psi_{n}|\dot{H}| \psi_{0}\right\rangle \ll$ $\left(E_{n}-E_{0}\right)^{2}$ and $\left\langle\psi_{n}|\ddot{H}| \psi_{0}\right\rangle \ll\left(E_{n}-E_{0}\right)^{3}$ etc. Here $e^{i \varphi_{n}}$ denotes a pure phase factor (including the Berry phase). However, this adiabaticity condition $\left\langle\psi_{n}|\dot{H}| \psi_{0}\right\rangle \ll$ $\left(E_{n}-E_{0}\right)^{2}$ obviously works for discrete spectra only. For infinitely large systems (thermodynamic limit) which support the propagation of gap-less Goldstone modes such as sound waves, for example, this condition does not apply. In this case, an external time-dependence typically induces non-equilibrium phenomena at some level (e.g., large wavelengths). As a result, the vacuum/ground state becomes ill-defined (i.e., non-unique) in these non-static scenarios leading to effects such as the creation of quasi-particles $\left|\psi_{n>0}\right\rangle$. Remembering the theory of quantum fields in curved space-times (see, e.g., [1]), we see that one encounters very similar problems and phenomena there. As we shall see later, there are indeed deep analogies between gravity and condensed matter, which allow us to describe their properties in a universal way and to understand many of these non-equilibrium phenomena in condensed matter in terms of geometrical concepts such as horizons.

Besides the above motivation from the condensed-matter side, the study of these analogies is also interesting from a (quantum) gravity point of view. In several important phenomena of quantum fields in curved space-times such as Hawking radiation or the quantum creation of the seeds for structure formation during inflation - the outgoing modes originate from initial quantum fluctuations at extremely high (trans-Planckian) energies/momenta, where the semi-classical treatment based on classical space-times is expected to break down (trans-Planckian problem). This observation poses the question of whether and how these effects depend on the microscopic structure at these ultra-high energies. Since we do not really know (yet) how to answer this question within a full theory of quantum gravity, conclusions by analogy to suitable condensed-matter systems - where we do understand the microscopic structure - might give us some hints.

Finally, condensed-matter theory provides us with a plethora of emergent phenomena, i.e., large-scale properties which are not part of the microscopic structure but emerge at low energies. For example, a large class of fluids with very different microscopic structures is described by the Euler equation at long length scales and low energies. Therefore, the study of condensed-matter analogues allows us to distinguish between universal (e.g., emergent) properties and system-specific features, which provide some information about the underlying microscopic structure.

\section{THE UNDERLYING ANALOGY}

Motivated by the aforementioned trans-Planckian problem, Bill Unruh suggested 2] studying sound waves in an irrotational and inviscid (i.e., frictionless) flow. In view of the vanishing vorticity $\boldsymbol{\nabla} \times \boldsymbol{v}=0$, sound perturbations can be described by a scalar potential via $\delta \boldsymbol{v}=\boldsymbol{\nabla} \phi$. Insertion into the Euler and continuity equa- 
tions yields the wave equation for sound

$$
\left(\frac{\partial}{\partial t}+\nabla \cdot \boldsymbol{v}_{0}\right) \frac{\varrho_{0}}{c_{\mathrm{S}}^{2}}\left(\frac{\partial}{\partial t}+\boldsymbol{v}_{0} \cdot \nabla\right) \phi=\nabla \cdot\left(\varrho_{0} \boldsymbol{\nabla} \phi\right)
$$

where $\varrho_{0}$ and $\boldsymbol{v}_{0}$ denote the density and velocity of the background flow and $c_{\mathrm{s}}$ is the speed of sound. Interestingly, this wave equation is identical to that of a scalar field $\phi$ in a curved space-time

$$
\square_{\mathrm{eff}} \phi=\frac{1}{\sqrt{-g_{\mathrm{eff}}}} \partial_{\mu}\left(\sqrt{-g_{\mathrm{eff}}} g_{\mathrm{eff}}^{\mu \nu} \partial_{\nu} \phi\right)=0,
$$

if we encode the properties of the background flow into the effective metric $g_{\mathrm{eff}}^{\mu \nu}$ in the Painlevé-GullstrandLemaître form [3]

$$
g_{\mathrm{eff}}^{\mu \nu}=\frac{1}{\varrho_{0} c_{\mathrm{s}}}\left(\begin{array}{cc}
1 & \boldsymbol{v}_{0} \\
\boldsymbol{v}_{0} & \boldsymbol{v}_{0} \otimes \boldsymbol{v}_{0}-c_{\mathrm{s}}^{2} \mathbf{1}
\end{array}\right) .
$$

In summary, we arrive at the qualitative analogy (sketched below) on the kinematic level - which does, however, not extend to the dynamics. [29] Of course, the background flow is governed by the Euler equation and thus the above metric is not obtained by solving the Einstein equations.

$$
\begin{aligned}
\text { Phonons (quantised) } & \leftrightarrow \text { Quantum fields } \\
\text { Fluid flow (classical) } & \leftrightarrow \text { Gravitational field } \\
\text { Euler equation } & \neq \text { Einstein equations }
\end{aligned}
$$

Having established this striking analogy between phonons in flowing fluids and (scalar) quantum fields in curved space-times - in spite of the in-equivalence of Euler and Einstein equations, one may wonder whether it is a peculiarity of sound waves or a more general phenomenon. As it turns out, the analogy can be extended to more general quasi-particles under appropriate conditions: The most general linearised low-energy effective action for scalar Goldstone-mode quasi-particles $\phi$ can be cast into the following form

$$
\mathcal{L}_{\text {eff }}=\frac{1}{2}\left(\partial_{\mu} \phi\right)\left(\partial_{\nu} \phi\right) G^{\mu \nu}(\underline{x})+\mathcal{O}\left(\phi^{3}\right)+\mathcal{O}\left(\partial^{3}\right),
$$

where $G^{\mu \nu}(\underline{x})$ denotes some tensor depending on the underlying condensed-matter system, see, e.g., [7, 8]. Higher-order contributions $\mathcal{O}\left(\phi^{3}\right)$ and $\mathcal{O}\left(\partial^{3}\right)$ have been omitted since we are considering a linearised and lowenergy effective action. Note that additional terms like $U^{\mu}(\underline{x}) \phi \partial_{\mu} \phi$ would be equivalent to a potential term $V(\underline{x}) \phi^{2}$ after an integration by parts - which is forbidden for Goldstone (i.e., gap-less) modes. (However, a potential term $V(\underline{x}) \phi^{2}$ would not spoil the curved space-time analogy and could be included as well - as long as it is still within the low-energy realm.) As usual, source terms like $J(\underline{x}) \phi$ and $K^{\mu}(\underline{x}) \partial_{\mu} \phi$ can be absorbed by a field-redefinition (quadratic completion).

Under these circumstances, we see that also more general quasi-particles $\phi$ are analogous to quantum fields in curved space-times. Again all relevant features of the underlying condensed-matter system are encoded in the tensor $G^{\mu \nu}$ and hence in the effective metric $G^{\mu \nu} \rightarrow$ $g_{\text {eff }}^{\mu \nu} \sqrt{-g_{\text {eff }}}$. Besides phonons, examples for such quasiparticles are ripplons (surface waves in fluids, see, e.g., 9|) and magnons (magnetisation variations, see, e.g., [10]), provided that they can be described by a single scalar field $\phi$. For multiple (non-scalar) quasi-particle modes $\phi_{a}$ which mix, the tensor $G^{\mu \nu}$ acquires additional indices in general $G_{a b}^{\mu \nu}$ and the analogy to an effective metric breaks down in the absence of additional symmetries. If these multiple quasi-particle modes are related via a symmetry, however, it may be possible to extend the curved space-time analogy to the non-scalar case. One example are photons in a dielectric medium with a constant permittivity $\varepsilon$ and a possibly space-time dependent four-velocity $u^{\mu}$, see [11] and 12]. In this case, both polarisations behave exactly as in a gravitational field described by the effective (Gordon) metric [13]

$$
g_{\mathrm{eff}}^{\mu \nu}=g_{\text {Minkowski }}^{\mu \nu}+(\varepsilon-1) u^{\mu} u^{\nu} .
$$

\section{EMERGENT HORIZONS}

Due to the qualitative analogy between phonons (or other quasi-particles) and quantum fields in curved space-times, all kinematic aspects of the phonons are encoded in the effective metric $g_{\text {eff }}^{\mu \nu}\left(\varrho_{0}, c_{\mathrm{s}}, \boldsymbol{v}_{0}\right)$. Consequently, this analogy provides a universal description (at low energies), i.e., we can forget all the microscopic details of the underlying fluid and apply the powerful tools and geometrical concepts known from general relativity 14] to this metric. For example, we may introduce sound cones (in analogy to light cones) via

$$
d s_{\text {eff }}^{2}=g_{\mu \nu}^{\mathrm{eff}} d x^{\mu} d x^{\nu}=0,
$$

which determine the causal structure of the fluid - i.e., which points can be connected by sound waves (or other quasi-particles) and which cannot. In general relativity, a break-down of causal connection (latter case) induced by the dynamics of the background (space-time) corresponds to the existence of a horizon - and this useful concept can now be transferred to condensed-matter systems, see also [15]. In order to be in equilibrium, every point of a condensed-matter system must be able to exchange energy with any other point (e.g., for equilibrating their local temperature or pressure). Since such an exchange is, at low energies, mediated by quasi-particles such as phonons, the emergence of a horizon analogue always indicates a departure from equilibrium and entails the amplification of quantum fluctuations (creation of quasi-particles) in general.

\section{EVENT HORIZON}

As a first example, let us consider the event horizon known from black holes. The event horizon is defined as the border of the region from where nothing is able to 
escape to infinity, cf. Fig. 1. Even though black holes are supposed to be completely black at the classical level, the incorporation of quantum effects suggests that they emit thermal radiation (black hole evaporation) as determined by the Hawking temperature [16]

$$
T_{\text {Hawking }}=\frac{1}{8 \pi M} \frac{\hbar c^{3}}{G_{\mathrm{N}} k_{\mathrm{B}}},
$$

where $M$ is the (ADM) mass of the black hole. The Hawking effect provides a striking confirmation of Bekenstein's concept of black hole thermodynamics [17]. Extending the second law of thermodynamics to black holes then requires assigning a non-vanishing entropy to the black hole $S_{\mathrm{BH}}=k_{\mathrm{B}} A /\left(4 L_{\text {Planck }}^{2}\right)$, which is given by the horizon area $A$ in units of the Planck length $L_{\text {Planck }}$ squared. Understanding the meaning and origin of this entropy is one of the important questions a theory of quantum gravity should answer.

However, there is a potential flaw in this picture: Tracing the outgoing particles of the Hawking radiation back in time (and thereby undoing the immense gravitational red-shift near the horizon), one finds that they originate from modes with ultra-short wavelengths, which are far below the Planck length $L_{\text {Planck }}$ for realistic parameters. However, at such ultra-short length scales, one would not trust the semi-classical treatment of quantum fields propagating in classical space-times anymore. Instead, effects of quantum gravity are expected to become important at such scales. Therefore, the derivation of Hawking radiation relies on the extrapolation of a theory into a region where it is expected to break down. Ergo, one arrives at the question of whether the Hawking effect is just an artifact of this unphysical over-extrapolation or whether it survives even after taking into account the proper microscopic structure (trans-Planckian problem).

\section{IMPACT OF DISPERSION RELATION}

Since we do not know (yet) the microscopic structure at the Planck scale (and beyond), it is very hard to answer this important question for real gravity. Fortunately, the analogy sketched in Fig. 1 yields a toy model which captures most of the relevant features and is still simple enough that we are able to do the calculations in certain cases. In view of the qualitative analogy in Sec. III one would also expect the de Laval nozzle to emit Hawking radiation: Even though the in-flowing fluid is at zero temperature, there should be a thermal flux of phonons (a faint hissing noise) escaping to the right-hand side. Repeating the same derivation for the metric (4) instead of the Schwarzschild geometry, the (analogue) Hawking temperature is determined by the velocity gradient at the horizon (where $v_{0}=c_{\mathrm{s}}$ ), see [2]

$$
T_{\text {Hawking }}=\frac{\hbar}{2 \pi k_{\mathrm{B}}}\left|\frac{\partial}{\partial r}\left(v_{0}-c_{\mathrm{S}}\right)\right| \text {. }
$$

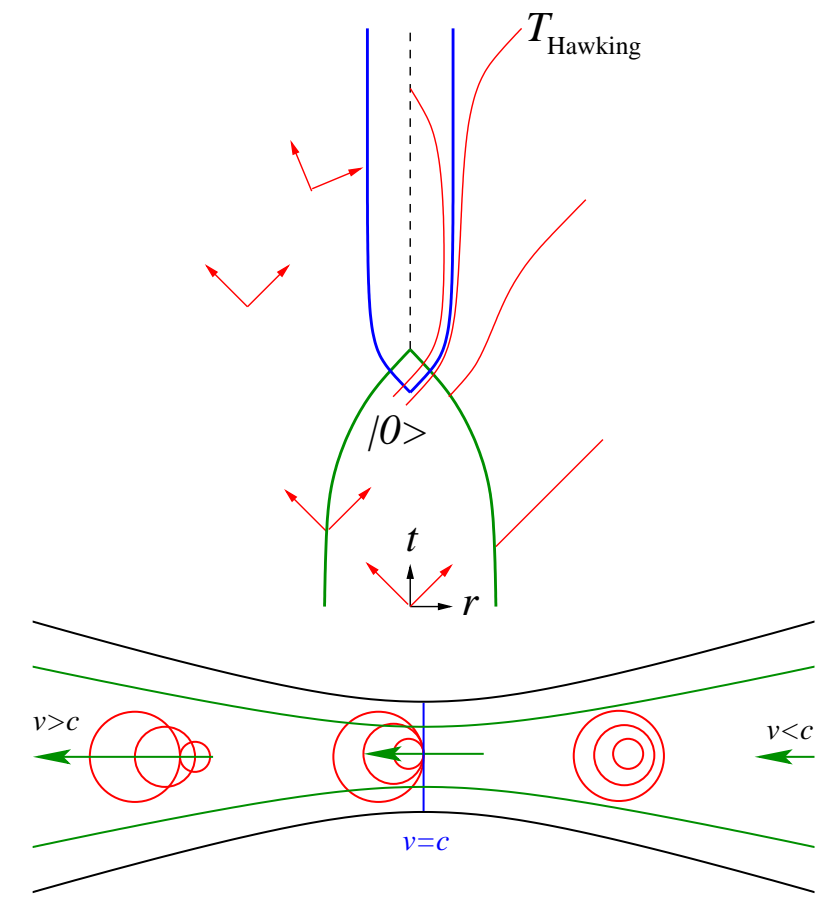

FIG. 1: Space-time diagram of the collapse of matter to a black hole (top) and sketch of a de Laval nozzle as the analogue of an event horizon (bottom). In the top picture, the green line indicates the surface of the collapsing matter and the black dashed line is the singularity. Light rays are denoted by red lines/arrows. The event horizon (blue line) lies between the last light ray which is able to escape to infinity and the first ray which is trapped. In the fluid analogue (bottom), green lines/arrows denote the fluid flow and the black lines are the walls of the nozzle. At its entrance, the flow is sub-sonic; and the flow velocity reaches the speed of sound at the narrowest point. Somewhat against intuition, the flow accelerates further (instead of slowing down) when the nozzle becomes wider again (as can be seen from the Bernoulli equation) and exits the nozzle with super-sonic speed. Sound waves (red circles) cannot escape from the super-sonic to the sub-sonic region and thus the boundary (blue line) between the two is completely analogous to an event horizon.

Depending on the experimental realisation, this temperature could range from a few nano-Kelvin (for BoseEinstein condensates, see, e.g., [18, 19]) up to fractions of a Kelvin (e.g., for wave-guides, cf. [20]). These values are much larger than the Hawking temperatures of real solar-size black holes, but still very hard to measure [21]. Besides a possible experimental realisation, we may also use the fluid analogues as toy models for quantum gravity in order to address the trans-Planckian problem. Again, the outgoing long-wavelength phonons originate from short-wavelength modes near the horizon - for which the fluid description breaks down. Fortunately, we understand the microscopic structure of fluids much better than quantum gravity. For many fluids, the first deviations from the Euler equation at short distances manifest themselves in a change of the dispersion relation for sound. In the case of Bose-Einstein condensates, for 
example, the dispersion relation reads (for a fluid at rest)

$$
\omega^{2}=c_{\mathrm{s}}^{2} k^{2}+\frac{\hbar^{2} k^{4}}{4 m^{2}},
$$

where $m$ is the mass of the condensed particles 22]. (Note that this quantity does not appear at all in the Euler equation.) For large wave-numbers $k$, the dispersion relation approaches the non-relativistic free-particle limit $E=\hbar^{2} k^{2} /(2 m)$ and thus becomes super-sonic, i.e., group and phase velocity exceed the speed of sound $v_{\text {group }}=d \omega / d k>v_{\text {phase }}=\omega / k>c_{\mathrm{s}}$. Hence, a wavepacket with such large wave-numbers is able to overcome the frame-dragging with the flow velocity $v$ and thereby to approach the sonic horizon from the inside, see Fig. 2. During that process, the inhomogeneity of $v$ (i.e., $v$ is smaller on the front end of the wave-packet than on its rear end) stretches the wave-packet and reduces its wavenumber (analogous to the gravitational red-shift) and thereby lowers its group velocity. Eventually, the wavepacket gets "ripped apart" and one part (the Hawking radiation) escapes into the exterior region whereas the remaining part (the in-falling partner particle) is swept away into the interior domain. Assuming that the wavepacket was in its ground state initially (for large $k$ ), the combined quantum state of the outgoing Hawking quanta and their in-falling partner particles is a pure state but the reduced density matrix of the Hawking radiation alone is thermal with the Hawking temperature, provided that the knee frequency, i.e., the frequency where the dispersion relation deviates from the linear behaviour (the analogue of the Planck scale), is much bigger than the Hawking temperature, see., e.g., [23, 24].

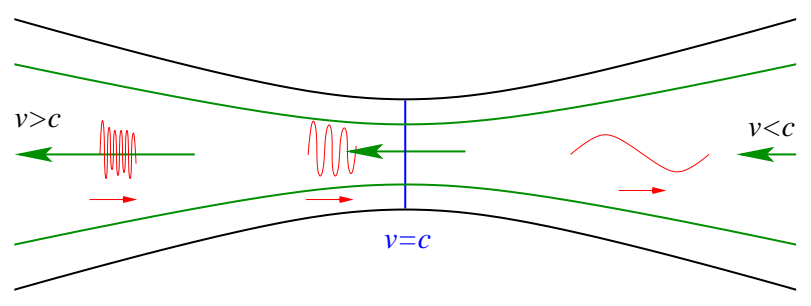

FIG. 2: Sketch of the origin of Hawking radiation (red curves) in a de Laval nozzle.

These considerations can be generalised to almost arbitrary dispersion relations (including the sub-sonic case [30]) and show that the Hawking effect survives in a large class of systems for which the dispersion relation $\omega(k)$ behaves regularly 31 for all $k$ and becomes linear $\omega=c_{\mathrm{s}} k$ at the typical frequency of the outgoing Hawking radiation. However, so far everything referred to the local fluid frame - which is analogous to the local freely falling frame in gravity. For example, the frequency $\omega$ in Eq. (10) is measured with respect to the co-moving fluid frame. The (conserved) frequency $\Omega$ in the laboratory frame - which would correspond to the global rest frame of the black hole in gravity - is locally Doppler shifted
$(\Omega+v k)^{2}=\omega^{2}(k)$. Now, if we allow for some interaction between the fluid and the walls of the nozzle (which are static in the laboratory frame), we would include an effective friction term $\gamma$ into this dispersion relation

$$
(\Omega+v k)^{2}+i \gamma \Omega=\omega^{2}(k) .
$$

Calculating the solutions of this quadratic equation, we see that the frequency $\Omega$ acquires an imaginary part which is proportional to $\gamma$ multiplied by the difference between the flow velocity $v_{\text {fluid }}$ and the sound speed (or, more precisely, its phase velocity $\left.v_{\text {phase }}=\omega / k\right)$. Therefore, for sub-sonic flow velocities, the interaction with the wall would just induce a damping of the sound waves (as one would expect). For super-sonic flow velocities, however, the effect reverses its sign and thus the phonons are not damped but amplified! This phenomenon is known as Miles instability [25] and is mainly responsible for the generation of water waves by wind. 32] This amplification mechanism may occur in the left half of the de Laval nozzle in Fig. 2] where the flow velocity exceeds the sound speed (analogous to the interior region of the black hole). Consequently, even if the wave-packet in Fig. 2 started out in its ground state (with respect to the fluid frame) for large $k$, a coupling to the wall would generate excitations on its way towards the horizon (due to $v_{\text {fluid }} \approx v_{\text {group }}>v_{\text {phase }}$ ) and these alterations of the initial quantum state would cause deviations from Hawking's result. For example, if the interaction with the wall causes the transition of the wave-packet from its ground state with respect to the fluid frame towards its ground state with respect to the rest frame of the walls (let us assume fermionic quasi-particles for the moment in order to make the Bogoliubov coefficients $\left|\alpha_{k}^{2}\right|+\left|\beta_{k}^{2}\right|=1$ and single-mode Hamiltonians bounded from above and below), there would be no Hawking radiation at all (Boulware state).

\section{APPARENT HORIZON}

Since event horizons are coordinate and observer independent, they can only occur in inhomogeneous systems. In cosmology, on the other hand, other horizon concepts are more suitable for describing the loss of causal connection within an expanding universe. Recalling the analogy described in Section III, it turns out that an expanding universe can be modelled by a homogeneously and isotropically expanding fluid, see Fig. 3. In this situation, we may specify the effective metric in Eq. (4)

$$
d s_{\text {eff }}^{2}=\frac{\varrho_{0}}{c_{\mathrm{S}}}\left(\left[c_{s}^{2}-\boldsymbol{v}_{0}^{2}\right] d t^{2}+2 \boldsymbol{v}_{0} \cdot d \boldsymbol{r} d t-d \boldsymbol{r}^{2}\right),
$$

via inserting $\varrho_{0}(t, \boldsymbol{r}) \rightarrow \varrho_{0}(t)$ and hence $c_{\mathrm{S}}(t, \boldsymbol{r}) \rightarrow c_{\mathrm{S}}(t)$ [homogeneity] as well as $\boldsymbol{v}(t, \boldsymbol{r}) \rightarrow a(t) \boldsymbol{r}$ [isotropy]. Diagonalising the above metric by the transformation to co-moving coordinates via $\boldsymbol{r}=b(t) \boldsymbol{R}$, where $a=\dot{b} / b$, we arrive at the usual Friedmann-Robertson-Walker metric

$$
d s_{\mathrm{eff}}^{2}=\frac{\varrho_{0}(t)}{c_{\mathrm{S}}(t)}\left(c_{s}^{2}(t) d t^{2}-b^{2}(t) d \boldsymbol{R}^{2}\right) .
$$


For a static fluid, every point may send/receive sound waves to/from any other point. However, if the fluid is expanding such that its flow velocity exceeds the speed of sound at some point, this is obviously no longer true, see Fig. 3 .

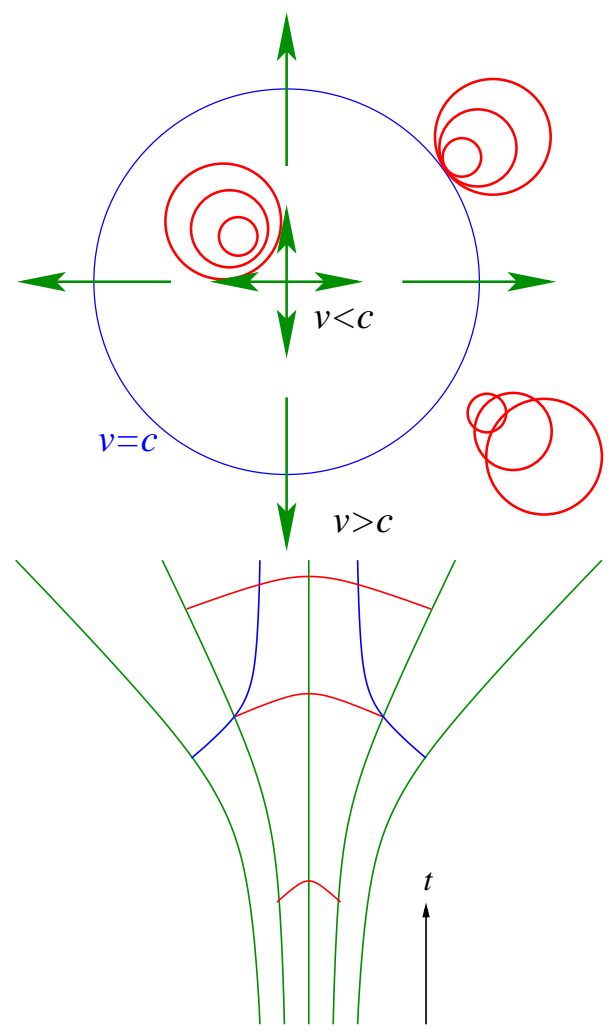

FIG. 3: Sketch of an instantaneous snap-shot (top) and spacetime diagram (bottom) of an expanding fluid. The green lines (bottom) denote fluid particle trajectories and the green arrows (top) the associated flow velocities (measured with respect to the centre of the fluid, which is at rest). The apparent horizon (blue) occurs where these flow velocities exceed the speed of sound. Obviously, no sound wave (red circles) can reach the centre from the region outside (at that instant of time). The three stages of the evolution of the sound modes (red lines) are sketched from bottom to top in the lower picture: Initially, they lie far within the horizon and hence oscillate almost freely. Later, they are stretched by the expansion of the fluid and cross the horizon. Afterwards, crest and trough of the wave are separated by the horizon and hence cannot exchange energy anymore, i.e., the oscillation freezes. As a result, the quantum state of this phonon mode cannot adapt to the expansion anymore and behaves non-adiabatically (amplification of quantum fluctuations by squeezing).

Employing the analogy to gravity, this border-line corresponds to an apparent horizon (i.e., the outermost trapped surface), which denotes the momentary loss of causal connection. In view of the quantitative correspondence described in Section [II, the initial quantum fluctuations of the phonon modes are frozen and amplified by the background expansion - in complete analogy to gravity. In our standard model of cosmology, precisely this mechanism (oscillation $\rightarrow$ horizon crossing $\rightarrow$ freezing $\rightarrow$ squeezing) is responsible for the generation of the seeds for structure formation out of the initial quantum fluctuations of the inflaton field during inflation. Traces of these frozen fluctuations can still be observed today in the anisotropies (on the level of $10^{-5}$ ) of the cosmic microwave background radiation. As demonstrated above, the same effect occurs in expanding fluids such as BoseEinstein condensates, see, e.g., [26]. In this case, the amplified quantum fluctuations result in small inhomogeneities, which are on the percent-level for realistic parameters (i.e., the generated contrast is even bigger than in the cosmological case) and could become measurable in near-future experiments. Besides the experimental point of view, one may use this analogy to study the impact of the dispersion relation etc.

\section{PARTICLE HORIZON}

Recalling the effective metric in Eq. (4), we see that an expanding/contracting universe can also be modelled by a fluid at rest $\boldsymbol{v}_{0}=0$ - provided that the speed of sound changes with time

$$
d s_{\mathrm{eff}}^{2}=\varrho_{0} c_{\mathrm{s}}(t) d t^{2}-\frac{\varrho_{0}}{c_{\mathrm{s}}(t)} d \boldsymbol{r}^{2} .
$$

In this case, an apparent horizon (which depends on the chosen time slicing) cannot be defined with respect to the laboratory time $t$ and, therefore, yet another horizon concept is more useful. If the speed of sound decreases fast enough, sound waves can only travel a finite distance, see Fig. VII

$$
r_{\text {horizon }}(t)=\int_{t}^{\infty} d t^{\prime} c_{\mathrm{s}}\left(t^{\prime}\right),
$$

and a particle horizon arises. Since the effective metrics in Eqs. (12) and (14) are related to each other [for suitable dynamics $b(t)$ and $\left.c_{\mathrm{s}}(t)\right]$ via a simple coordinate transformation, they describe the same physical effects - even though the underlying condensed-matter systems are very different (expanding fluid vs. fluid at rest). This observation demonstrates the universality of the geometrical concepts and allows us to predict the same amplification mechanism (oscillation $\rightarrow$ horizon crossing $\rightarrow$ freezing $\rightarrow$ squeezing) as in the previous Section. For a fluid at rest, the wavelength of a given mode remains constant, but the size of the particle horizon shrinks constantly [as can be seen from Eq. (15)] and thus every mode crosses the horizon at some time, see Fig. VII

A prototypical example for a condensed-matter system in which the speed of sound (or other quasi-particles) goes down to zero is a sweep through a zero-temperature phase transition by means of a time-dependent external parameter $g(t)$, see Fig. VII] With the same assumptions as in Eq. (5), the effective Hamiltonian density for the 


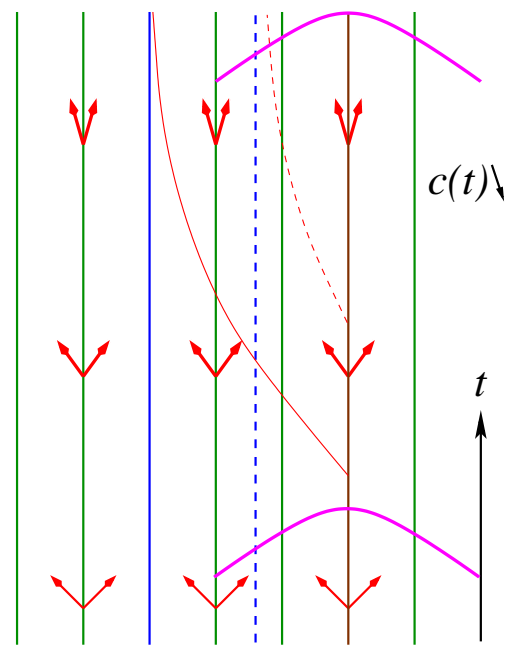

FIG. 4: Sketch of the space-time diagram of the sweep through such a phase transition and the emergence of a particle horizon. Again, the green lines denote fluid particle trajectories and the red arrows are the time-dependent sound cones. If the speed of sound decreases fast enough, a sound wave (red curve) can only travel a finite distance and hence the region beyond the particle horizon (blue line) is causally disconnected from the brown trajectory. Since the size of the particle horizon shrinks constantly (see dashed red curve and dashed blue line for a later time), every sound mode (magenta curve) crosses the horizon at some instant and freezes.

quasi-particle excitations $\phi$ within the homogeneous and isotropic sample can be cast into the general form

$$
\mathcal{H}=\frac{1}{2}\left(\alpha \Pi^{2}+\beta(\nabla \phi)^{2}\right),
$$

where $\Pi$ is the canonically conjugate momentum density and $\alpha$ and $\beta$ are external parameters which depend on $g(t)$ and thereby on time. If the quasi-particle excitations $\phi$ are stable before the transition $g<g_{c}$ (i.e., $\alpha, \beta>0)$ but become unstable after crossing the critical point $g>g_{c}$ (i.e., they trigger the decay of the false vacuum down to the real ground state), at least one of the two parameters must change its sign at $g=g_{c}$. Consequently, the speed of sound $c_{\mathrm{s}}^{2}=\alpha \beta$ goes to zero at the transition (and becomes imaginary afterwards - indicating the aforementioned instability), i.e., the $\phi$-modes become arbitrarily soft when approaching the transition. The frozen and amplified quantum fluctuations (as long as they are in the linear low-energy regime) can then be calculated in complete analogy to cosmology 10, 27]. The other way around, one might use these condensedmatter analogues as toy models for quantum gravity in order to address questions such as the quantum backreaction problem or possible low-energy signatures of Planck-scale physics, see, e.g., 28].

\section{LESSONS FOR QUANTUM GRAVITY?}

What are the lessons for quantum gravity to be learned from these analogies? We found that the Hawking effect - even though its original derivation is based on an overextrapolation of the semi-classical analysis - seems to be fairly robust against alterations of the physics at ultrahigh (Planckian) energies for a large class of models. On the other hand, there are also physically reasonable examples which display strong deviations from Hawking's prediction. Pictorially speaking, it appears to be important whether the "space-time foam" of quantum gravity is freely falling into the black hole (as everything else) or whether it is fixed with respect to the global rest frame of the black hole and just "tells" the matter to fall down. In the first case, one would expect 33 Hawking's prediction to be valid (if the microscopic behaviour is not too violent, i.e., adiabatic); whereas, in the second situation, one could easily imagine deviations from Hawking's result.

Furthermore, we observed that the Hawking effect is fairly independent of the Einstein equations and just requires the existence of an emergent (event) horizon. The concept of black-hole entropy, on the other hand, cannot be applied to the sonic analogues and probably relies on some features of the Einstein equations. In the light of this observation, the frequently suggested interpretation of the (non-universal) black-hole entropy via the entanglement of the (universal) Hawking radiation appears a bit dubious.

In contrast to effective metrics and horizons, which emerge quite naturally in many condensed matter systems, it is very hard to reproduce the principle of equivalence, which might give us some hints about the underlying structure of quantum gravity. Speculating a bit further, one may ask whether every microscopic model which incorporates general covariance in a suitable form would lead to the Einstein equations as low-energy effective theory and hence constitute a valid candidate for quantum gravity. (Demanding covariance on a microscopic level could perhaps explain why the direct search for Lorentz violations was negative so far.) As we know from condensed matter, many fluids with totally different microscopic structures are described by the same Euler equation at large distances and low energies - the same could perhaps be true for the Einstein equations.

\section{Acknowledgments}

R. S. acknowledges valuable discussions with Bill Unruh and many others as well as financial support by the Emmy-Noether Programme of the German Research Foundation (DFG, SCHU 1557/1-2,3).
[1] N. D. Birrell and P. C. W. Davies, Quantum Fields in Curved Space (Cambridge University Press, Cambridge,
England 1982); 
S. A. Fulling, Aspects of Quantum Field Theory in Curved Space-Time (Cambridge University Press, Cambridge, England 1989).

[2] W. G. Unruh, Phys. Rev. Lett. 46 (1981) 1351; Phys. Rev. D 51 (1995) 2827.

[3] P. Painlevé, C. R. Hebd. Seances Acad. Sci. (Paris) 173 (1921) 677;

A. Gullstrand, Ark. Mat. Astron. Fys. 16 (1922) 1;

G. Lemaître, Ann. Soc. Sci. (Bruxelles) A 53 (1933) 51.

[4] P. O. Fedichev and U. R. Fischer, Phys. Rev. Lett. 91 (2003) 240407;

Phys. Rev. D 69 (2004) 064021.

[5] W. G. Unruh, Phys. Rev. D 14 (1976) 870.

[6] R. Schützhold, G. Schaller, and D. Habs, Phys. Rev. Lett. 97 (2006) 121302; see also A. Retzker, J. I. Cirac, M. B. Plenio, and B. Reznik, arXiv:0709.2425.

[7] C. Barceló, S. Liberati, and M. Visser, Analogue Gravity, Living Rev. Rel. 8 (2005) 12; and references therein; as well as G. E. Volovik, Universe in a Helium Droplet (Oxford University Press, Oxford, 2003); and references therein.

[8] C. Barcelo, S. Liberati and M. Visser, Class. Quant. Grav. 18 (2001) 3595; ibid 19 (2002) 2961; Int. J. Mod. Phys. D 10 (2001) 799.

[9] R. Schützhold and W. G. Unruh, Phys. Rev. D 66 (2002) 044019.

[10] R. Schützhold, Phys. Rev. Lett. 95 (2005) 135703.

[11] R. Schützhold, G. Plunien, and G. Soff, Phys. Rev. Lett. 88 (2002) 061101.

[12] W. G. Unruh and R. Schützhold, Phys. Rev. D 68 (2003) 024008; see also U. Leonhardt and P. Piwnicki, Phys. Rev. Lett. 84 (2000) 822; with comment by M. Visser, ibid. 85 (2000) 5252; and reply U. Leonhardt and P. Piwnicki, ibid. 85 (2000) 5253.

[13] W. Gordon, Ann. Phys. (Leipzig) 72 (1923) 421.

[14] C. M. Misner, K. S. Thorne, and J. A. Wheeler, Gravitation (Freemann, San Francisco, 1973); S. W. Hawking and G. F. R. Ellis, The Large Scale Structure of Spacetime (Cambridge University Press, Cambridge, England, 1973).

[15] R. Schützhold and M. Uhlmann, Horizon Analogues in the Laboratory, Proceedings of the Memorial Symposium for Gerhard Soff (April 25 and 26, 2005, Frankfurt, Germany); R. Schützhold and W. G. Unruh (eds.), Quantum Analogues: From Phase Transitions to Black Holes 65 Cosmology, Springer Lecture Notes in Physics 718 (2007).

[16] S. W. Hawking, Nature 248 (1974) 30; Commun. Math. Phys. 43 (1975) 199.

[17] J. D. Bekenstein, Lett. Nuovo Cim. 4 (1972) 737; Phys. Rev. D 7 (1973) 2333; ibid 9 (1974) 3292; ibid 12 (1975) 3077; J. M. Bardeen, B. Carter and S. W. Hawking, Commun. Math. Phys. 31 (1973) 161.

[18] L. J. Garay, J. R. Anglin, J. I. Cirac, and P. Zoller, Phys. Rev. Lett. 85 (2000) 4643; Phys. Rev. A 63 (2001) 023611; S. Giovanazzi, C. Farrell, T. Kiss, and U. Leonhardt, Phys. Rev. A 70 (2004) 063602.

[19] R. Schützhold, Phys. Rev. Lett. 97 (2006) 190405.

[20] R. Schützhold and W. G. Unruh, Phys. Rev. Lett. 95 (2005) 031301.

[21] W. G. Unruh, Measurability of Dumb Hole Radiation?, in
M. Novello, M. Visser, and G. Volovik (editors), Artificial Black Holes (World Scientific, Singapore, 2002).

[22] F. Dalfovo, S. Giorgini, L. P. Pitaevskii, and S. Stringari, Rev. Mod. Phys. 71 (1999) 463; A. J. Leggett, ibid 73 (2001) 307.

[23] W. G. Unruh and R. Schützhold, Phys. Rev. D 71 (2005) 024028.

[24] T. Jacobson, Prog. Theor. Phys. Suppl. 136 (1999) 1; Phys. Rev. D 44 (1991) 1731; ibid 48 (1993) 728; ibid 53 (1996) 7082; T. Jacobson and D. Mattingly, ibid 61 (2000) 024017; S. Corley and T. Jacobson, ibid 57 (1998) 6269; ibid 59 (1999) 124011; S. Corley, ibid 55 (1997) 6155; ibid 57 (1998) 6280; R. Brout, S. Massar, R. Parentani and P. Spindel, ibid 52 (1995) 4559.

[25] J. W. Miles, J. Fluid Mech. 3 (1957) 185; G. E. Vekstein, Am. J. Phys. 66 (1998) 886.

[26] M. Uhlmann, Y. Xu, and R. Schützhold, New J. Phys. 7 (2005) 248;

Ralf Schützhold et al, Phys. Rev. Lett. 99 (2007) 201301.

[27] U. R. Fischer and R. Schützhold, Phys. Rev. A 70 (2004) 063615; R. Schützhold, M. Uhlmann, Y. Xu and U. R. Fischer, Phys. Rev. Lett. 97 (2006) 200601; Michael Uhlmann, Ralf Schützhold, and Uwe R. Fischer, Phys. Rev. Lett. 99 (2007) 120407.

[28] F. Queisser, M. Uhlmann, and R. Schützhold, Class. Quantum Grav. 24 (2007) 1375; R. Schützhold, M. Uhlmann, Y. Xu, and U. R. Fischer, Phys. Rev. D 72 (2005) 105005; C. Maia and R. Schützhold, ibid 76 (2007) $101502(\mathrm{R})$.

[29] Note that only the phonons "feel" the effective metric (4). A detector immersed in the fluid will evolve according to the Minkowski metric (unless it is designed in a special way, see, e.g., [4]). Therefore, the internal time of the detector will be the laboratory time and hence there will be no direct fluid analogue of the Unruh effect [5], as this effect is a result of the relativistic time dilatation with a constantly changing velocity and hence Lorentz boost factor. Nevertheless, it is possible to design analogues for indirect signatures of the Unruh effect, see, e.g., [6].

[30] For a sub-sonic dispersion relation $d \omega / d k<c_{\mathrm{s}}$, the initial short-wavelength wave-packet originates from the exterior region (r.h.s. in Fig. 2) where $v<c_{\mathrm{s}}$. However, since its group velocity is too small, it is swept towards the horizon and thereby stretched. Eventually, it also gets "ripped apart" into two final long-wavelength wavepackets, which are basically the same as in the supersonic case.

[31] Such as Eq. (10) or $\omega^{2}=c_{\mathrm{s}}^{2} k^{2} /\left(1+\eta^{2} k^{2}\right)$; but not $\omega^{2}=c_{\mathrm{s}}^{2} k^{2} /\left(1-\eta^{2} k^{2}\right)$, which becomes singular at $k=1 / \eta$ [manuscript in preparation].

[32] If the wind blows faster than the phase velocity of the water waves, they are not damped but amplified by their friction with air. Since deep-water waves with large wavelengths are faster than those with shorter wavelengths, the velocity of the wind determines the maximum size of the generated waves.

[33] But then one should worry what happens with the "space-time foam" at the singularity - which might be related to the mode-generation problem in cosmology and the black-hole information paradox. 